\title{
Structural investigations of T854A mutation in EGFR and identification of novel inhibitors using structure activity relationships
}

\author{
Sukriti Goyal', Salma Jamal', Asheesh Shanker', Abhinav Grover ${ }^{2 *}$ \\ From X-meeting 2014 - International Conference on the Brazilian Association for Bioinformatics and \\ Computational Biology \\ Belo Horizonte, Brazil. 28-30 October 2014
}

\begin{abstract}
Background: The epidermal growth factor receptor (EGFR) is a member of the ErbB family that is involved in a number of processes responsible for cancer development and progression such as angiogenesis, apoptosis, cell proliferation and metastatic spread. Malfunction in activation of protein tyrosine kinases has been shown to result in uncontrolled cell growth. The EGFR TK domain has been identified as suitable target in cancer therapy and tyrosine kinase inhibitors such as erlotinib have been used for treatment of cancer. Mutations in the region of the EGFR gene encoding the tyrosine kinase (TK) domain causes altered responses to EGFR TK inhibitors (TKI). In this paper we perform molecular dynamics simulations and PCA analysis on wild-type and mutant (T854A) structures to gain insight into the structural changes observed in the target protein upon mutation. We also report two novel inhibitors identified by combined approach of QSAR model development.

Results: The wild-type and mutant structure was observed to be stable for 26 ns and 24 ns respectively. In PCA analysis, the mutant structure proved to be more flexible than wild-type. We developed a 3D-QSAR model using 38 thiazolyl-pyrazoline compounds which was later used for prediction of inhibitory activity of natural compounds of ZINC library. The 3D-QSAR model was proved to be robust by the statistical parameters such as $r^{2}(0.9751)$, $q^{2}(0.9491)$ and pred_r ${ }^{2}(0.9525)$.

Conclusion: Analysis of molecular dynamics simulations results indicate stability loss and increased flexibility in the mutant structure. This flexibility results in structural changes which render the mutant protein drug resistant against erlotinib. We report two novel compounds having high predicted inhibitory activity to EGFR TK domain with both wild-type and mutant structure.
\end{abstract}

\section{Introduction}

The epidermal growth factor receptor (EGFR) is a member of the ErbB family that is involved in a number of processes responsible for cancer development and progression such as angiogenesis, apoptosis, cell proliferation and metastatic spread [1]. The EGFR family comprises of four receptors namely, EGFR (ErbB1/HER1), ErbB2 (HER2/neu), ErbB3 (HER3) and ErbB4 (HER4). Various

\footnotetext{
* Correspondence: abhinavgr@gmail.com

${ }^{2}$ School of Biotechnology, Jawaharlal Nehru University, New Delhi, India 110067

Full list of author information is available at the end of the article
}

mechanisms including gene amplification and mutations result in a disturbed regulatory mechanism of EGFR signalling [2]. Malfunction in activation of such kinases has been shown to result in uncontrolled cell growth. The EGFR TK domain has been identified as suitable target in cancer therapy and drugs such as erlotinib have been used for treatment of cancer. However, mutations in the region of the EGFR gene encoding the tyrosine kinase (TK) domain causes altered responses to EGFR TK inhibitors (TKI) [2]. In 2004, these mutations were first identified in patients with non-small cell lung cancer (NSCLC)[1]. NSCLC of Caucasian origin account for 
$15 \%$ while NSCLC of Asian ethnicity for 30\% of EGFR mutations. These mutations are known to be associated with non-smoking status, adenocarcinoma histology and female gender $[3,4]$.

The most common mutations involve point mutations in exon 18 and exon 21, insertions or deletions in exon 19, insertions/duplications and point mutations in exon 20 [5]. Destabilization of equilibrium between the active and inactive state of EGFR kinase activity toward promoting enzyme activation is a result of these mutations which in turn causes EGFR to translate into tumor growth and gives a survival advantage [1,6]. Mutations T790M (gatekeeper), M766T (C helix), L718A (solvent chanel) and T854A (activation loop) are most common in erlotinib resistance [7]. For this study we will be studying a drugsensitive second-site EGFR mutation, T854A, which occurs due to change of Guanine in place of Adenine at nucleotide 2560 (exon 21)[8]. This non-synonymous single nucleotide polymorphism (SNP) results in substitution of Alanine for Threonine at position 854. The T854A residue is located at the bottom of the ATP binding site on C-lobe and its side chain is in contact distance of erlotinib or gefitinib. Thus, T854A substitution results in loss of contacts and binding affinity to these inhibitors.

In silico methodology for drug development is a viable and good option when compared to conventional drug development methods. One such in silico method involves development of quantitative structure activity relationship (QSAR) which establishes a correlation between the structure and inhibitory activity of molecular fragments of interests. 3D-QSAR is a robust technique in drug design process used to predict the inhibitory activities of the prospective lead compounds by applying the knowledge of three-dimensional properties of the lead compounds through a chemometric approach $[9,10]$. It develops models which indicate the synthesis of novel inhibitors assuming that the receptor binding ability is related to its inhibitory activity $[11,12]$. For development of QSAR model, the binding site of receptor is considered to be rigid and that the ligand molecules belong to a set of congeneric series [12]. Molecular fields including hydrophobic, steric and electrostatic interaction energies are calculated for the set of compounds. A molecular field analysis model is generated and evaluated for its robustness by calculation of statistical parameters.

In this study we performed molecular dynamics simulations on both wild-type (WT) and mutant (T854A) structures and analysed the structural changes [13-16]. A 3D-QSAR model was developed using 38 thiazolylpyrazoline derivatives reported by $\mathrm{Lv}$ et al (2011) against WT EGFR [17]. This model was then used to screen ZINC libraries for compounds with high predicted activity values which can be considered as lead drug candidates against both WT and mutant (T854A). This paper gives insights to the structural changes brought about by single nucleotide polymorphism in tyrosine kinase domain of EGFR. The compounds reported in this study can be considered for further experimental validation as potent lead compounds.

\section{Materials and methods}

\section{Generation of wild-type and mutant EGFR structures}

The crystal structure of WT EGFR was extracted from Protein Data Bank (PDB) [PDB ID: 4G5J][18]. The obtained crystal structure was first prepared using Protein preparation utility of Schrodinger [19-21]. Mutant structure was generated using Schrodinger Glide software through Maestro interface. The WT structure of EGFR was also subject to the identical in silico mutational method in which the wild-type residue (Threonine) was mutated to itself (Threonine) to ensure that mutation process is uniform for all structures. These structures were then subject to MD simulations.

\section{Molecular dynamics simulations}

The GROMACS package [22,23] was used for carrying out MD simulations using the same conditions as mentioned in previous works of our lab $[15,24,25]$. Comparative analysis of structural deviations in WT and mutated (T854A) structures of EGFR were carried out using Gromacs utility tools such as g_rmsf, g_rms, g_gyrate, g_sas etc.

\section{Principal component analysis}

The essential dynamics (ED) method was used for the computation of eigenvectors and eigenvalues with their projection along the first two principal components with the help of a protocol present in the GROMACS software package [26]. The principal component analysis (PCA) or ED is a protocol that simplifies the complexity of data obtained and extracts the important motion in MD that are significant for the biological function of the protein [26]. In this evaluation, a covariance matrix was created from the obtained trajectories once the rotational and translational movements were removed. The matrix was then diagonalized to identify a set of eigenvectors and eigenvalues. The amplitude of eigenvectors and the displacement of atoms along each of these eigenvectors show the concentrated motions of protein along each direction and were represented by eigenvalues. This analysis was performed using g_covar and g_anaeig of GROMACS utility tools.

\section{Selection and presentation of data set for QSAR model development}

The dataset of 45 thiazolyl-pyrazoline derivatives [30] and the template (Figure 1a) was drawn using Chemsketch (ACD/Chemsketch Freeware Version 12.01) These 


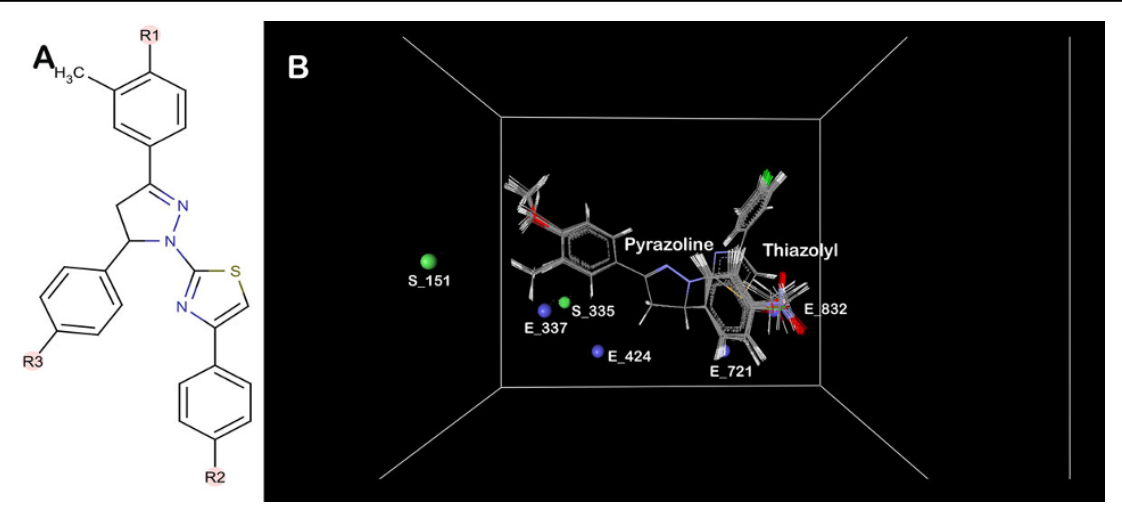

Figure 1 (a) Representation of structure of common template of thiazolyl-pyrazoline compounds. (b) Depiction of aligned set of molecules and 3D descriptors in cubic grid.

compounds were prepared by Vlife Engine module of Vlife MDS as described previously [9,27-29].

\section{Computation of force field}

The 38 thiazolyl-pyrazoline derivatives along with their pIC50 (negative logarithm of IC50) values (Table S1; additional file 1) were used for calculation of force field as described previously [9,27-29].

\section{Building the 3D-QSAR model of thiazolyl-pyrazoline derived compounds}

Sphere exclusion method was applied for division of 38 compounds comprising the dataset into training and test set. Stepwise forward multiple regression method was utilized using the advanced variable selection and model building wizard for development of the 3D-QSAR model with default values. The stepwise forward variable selection algorithm has been described in previous studies $[9,27,28,31]$.

\section{Validation of the developed 3D-QSAR model}

The integrity of the developed 3D-QSAR model was confirmed with the help of different statistical parameters including squared correlation coefficient $\left(\mathrm{r}^{2}\right)$, cross validated squared correlation coefficient $\left(\mathrm{q}^{2}\right)$, predicted squared correlation coefficient (pred_r $r^{2}$ ), F-test and standard error. The model is said to be robust if it has the following statistical parameters $\mathrm{r}^{2}>0.6, \mathrm{q}^{2}>0.6$ and pred_r ${ }^{2}>$ 0.5 [32-34]. The F-test is described as the variance observed by the developed QSAR model divided by the variance due to the error in the regression. Hence, statistical significance of the developed model can be explained with high F-test. The low standard error of Pred_r ${ }^{2}$ se, $q^{2}$ _se and $r^{2}{ }_{-}$se showed absolute fitness quality of the model.

\section{Model cross-validation}

The developed model was first validated internally and then externally as described previously $[9,27,28,31]$.
Briefly internal validation was carried out using the leave-one-out $\left(\mathrm{q}^{2}, \mathrm{LOO}\right)$ method while external validation involved prediction of inhibitory activity of each molecule comprising the test set by means of the QSAR model generated using compounds in the training set.

$\mathrm{Y}$ randomisation test was employed for examining the robustness of the developed models for training sets by calculation of Z-score as described previously $[9,27,28,31]$.

\section{Prediction of ZINC library using developed 3D-QSAR model}

A natural compound ZINC database containing 0.2 million compounds was prepared and used for prediction of inhibitory activity using the developed 3D-QSAR model. Compounds with high predicted inhibitory activity were selected for docking analysis.

\section{Docking of top scoring compounds with EGFR}

Docking of the top two compounds with WT and T854A structures was performed using the Glide module of Schrodinger [35,36] as described previously $[13,25,37]$.

\section{Results and discussion}

Structural and functional analysis of EGFR tyrosine kinase domain upon mutation

Molecular dynamics simulations for WT and mutant (T854A) EGFR TK protein was performed to gain insight into the structural and functional behaviour of the drug resistance associated mutation. We studied RMSD, RMSF, radius of gyration (Rg), solvent accessible surface area (SASA) and ED analysis between the WT and mutant (T854A) EGFR protein. RMSD for the backbone of the protein structures were calculated from the initial structure (Figure 2a). In this figure, till 14ns WT showed backbone RMSD of $\sim 0.13$ to $\sim 0.27 \mathrm{~nm}$ during this part of simulations. After $14 \mathrm{~ns}$ WT structure exhibited minimum deviation till the end of simulation 


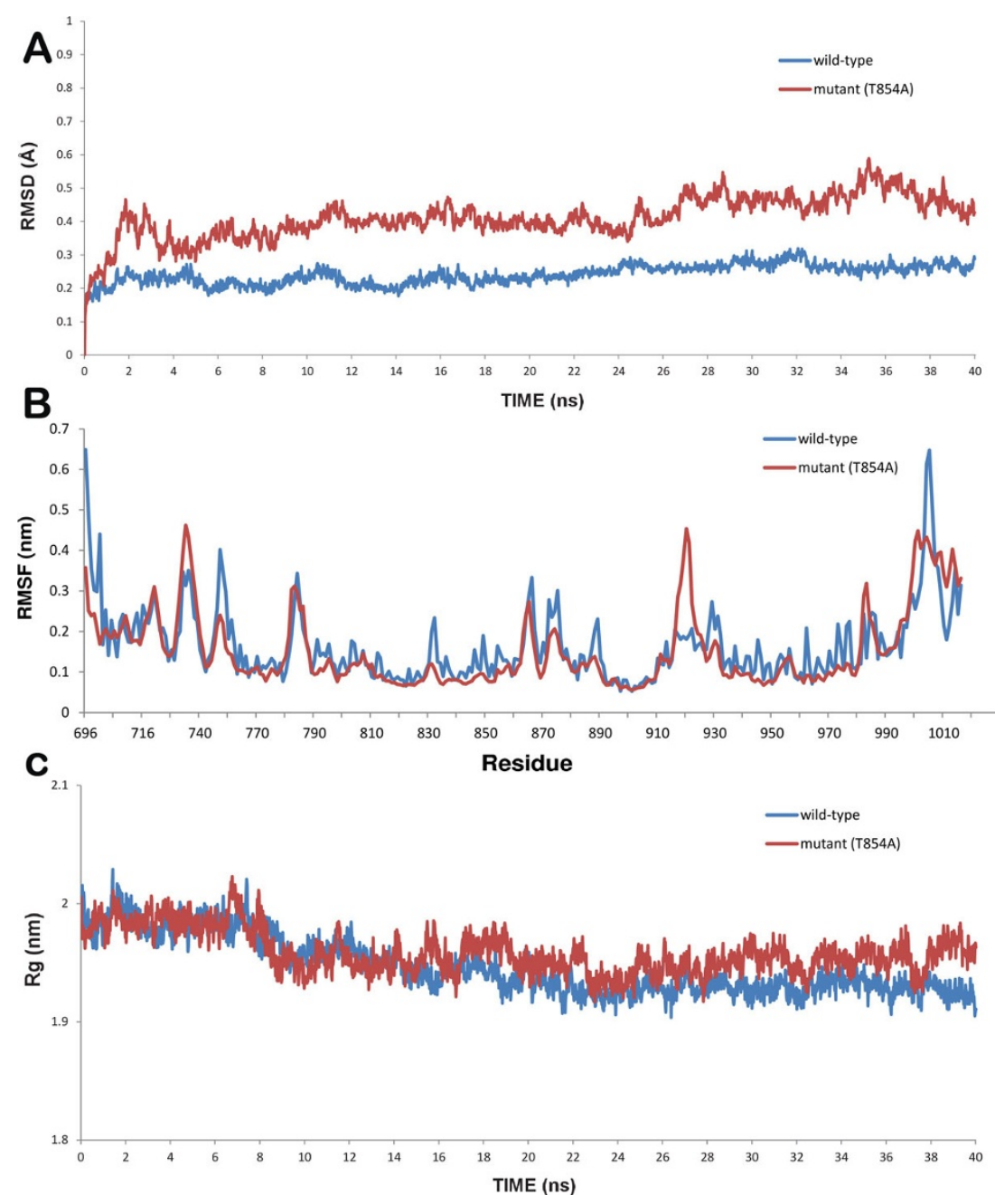

Figure 2 Graphs showing (a) RMSD (b) RMSF and (c) Radius of gyration of wild-type (blue) and mutant (T854A) (red) protein.

that is $40 \mathrm{~ns}$ with its backbone RMSD ranging from $\sim 0.14$ to $\sim 0.22 \mathrm{~nm}$, where as mutant structure showed maximum deviation till the end of simulation resulting backbone RMSD of $\sim 0.13$ to $\sim 0.47 \mathrm{~nm}$ respectively. This stable RMSD provided a suitable basis for further analysis. For determining the effect of mutation on the behaviour of residues, the RMSF values of WT and mutant (T854A) structures were calculated (Figure 2b). The results indicate higher degree of flexibility in mutant (T854A) in comparison to WT.

Another parameter radius of gyration ( $\mathrm{Rg}$ ) defined as the mass-weight root mean square distance of collection of atoms from their common centre of mass was helpful in giving further insight into the structural changes due to mutation and the overall dimension of the protein. Plot of radius of gyration of protein vs. time is shown in Figure 2c. It can be seen that mutant (T854A) structure exhibited higher Rg value in comparison to WT structure. Variation of SASA for both
WT and mutant (T854A) proteins with respect to time can be seen in Figure S1(a). WT structure was observed to have higher value of SASA with time, while mutant (T854A) showed lower value of SASA. Greater fluctuation in $\operatorname{Rg}$ in mutant (T854A) structure suggested structural alteration in the mutant structure. Since, hydrogen bonds play an important role in maintaining the stable conformation of protein, analysis of WT and mutant (T854A) proteins were performed with respect to time (Figure S1(b)). The total energy (Figure S1(c)) was observed to be more or less the same throughout the simulations for both WT and mutant (T854A).

All these results indicate that mutation (T854A) rendered the protein structure more flexible affecting the structural and functional behaviour of EGFR TK protein. This result was further validated by principal component analysis (PCA) analysis.

Essential dynamics (ED) analysis gives an improved analysis of dynamical mechanical properties of the protein 
system. To further support our MD simulations results, the large-scale collective motions of the WT and mutant (T854A) protein using ED analysis were determined. Principal components are the eigenvectors of a covariance matrix. This projection gives the change of particular trajectory along each eigenvector. The range of the corresponding eigenvalues (Figure 3) indicated that the fluctuation of the protein system was basically restricted to the first two eigenvectors. The motion of the two proteins in phase space can be shown by the projection of trajectories obtained at $300 \mathrm{~K}$ onto the first two principal components (PC1, PC2) in which we observe clusters of stable states. Analysis of these plots reveals that the clusters are well defined in WT than mutant (T854A). Also, mutant (T854A) covers a greater region of phase space mainly along PC1 plane than WT. It can thus be said that mutant $(\mathrm{T} 854 \mathrm{~A})$ is more flexible than WT at $300 \mathrm{~K}$. The values for trace of the diagonalized covariance matrix of the $\mathrm{C} \alpha$ atomic positional fluctuations obtained for WT protein and mutant $(\mathrm{T} 854 \mathrm{~A})$ protein were $7.603 \mathrm{~nm}^{2}$ and $18.3734 \mathrm{~nm}^{2}$ respectively. Trace is the total variance of the

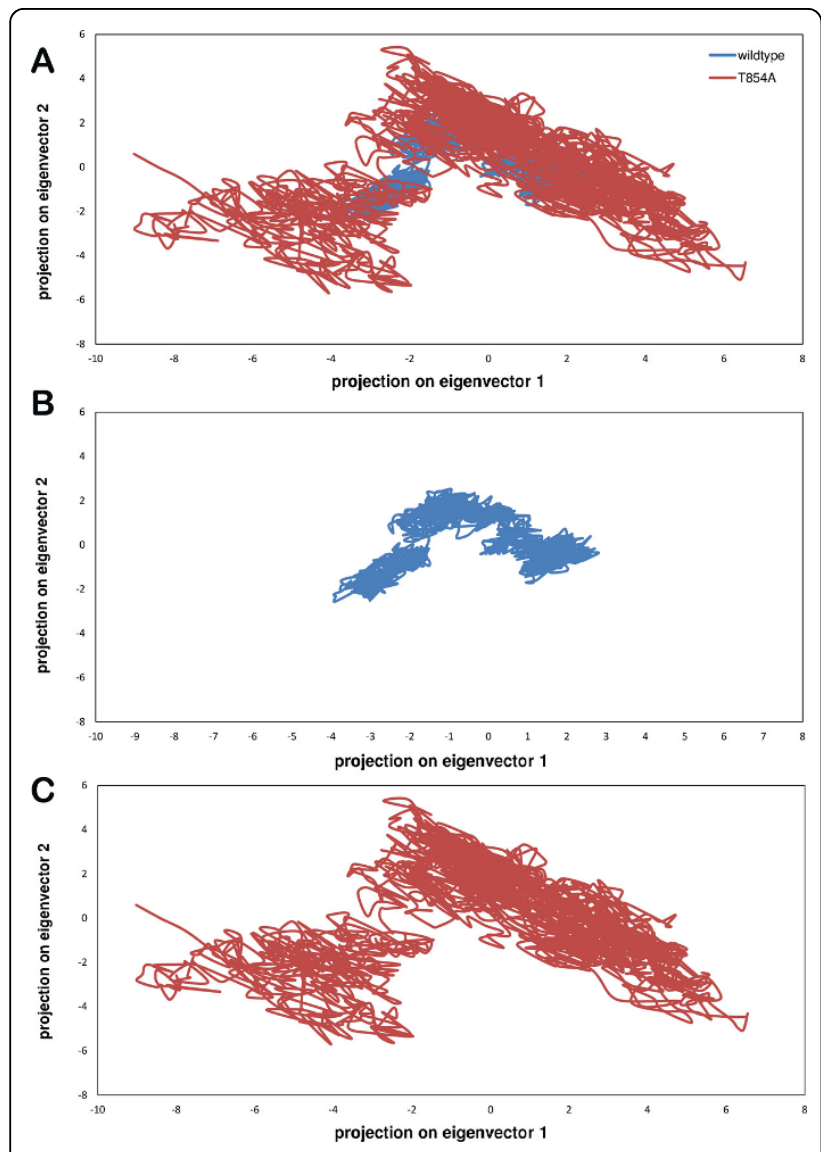

Figure 3 (a) Projection of the motion of the protein in phase space along the first two principal eigenvectors of wild-type (blue) and mutant (T854A) (red) protein structures. dataset thus again confirming the overall increased flexibility of mutant than WT at $300 \mathrm{~K}$.

Also it can be seen in Figure 4, T854A mutation causes change in active site. Since, 854 lies in the contact region of erlotinib but not close to ATP, this mutation results in reduced affinity for erlotinib while maintaining its kinase activity. Substitution of alanine in place of threonine causes the binding surface to move away from erlotinib indicating a possible reason for its decreased binding affinity. This mutation causes flexibility in the binding region of erlotinib while not affecting binding of ATP thus explaining its acquired resistance and maintained functionality.

\section{D QSAR model data selection}

A 3D-QSAR model development gives a statistical relationship between the structures and activity of chemical compounds by calculation of 3D molecular descriptors involving steric, electrostatic and hydrophobic points marked on the 3D spatial grid. The invariable columns were removed after computing the force field grid descriptors which resulted in 3163 descriptors from 3268 descriptors, thus removing 105 invariable descriptors. For development of the QSAR model, pIC50 was chosen as the dependent variable while the calculated 3D descriptors as independent variable. Division of dataset resulted in 11 compounds in test set while the rest 27 compounds in training set. The test set consisted of compounds 6,9 , $12,28,29,32,36,37,40,44$ and 45.

\section{D-QSAR model development and validation}

Stepwise forward (SW) multiple regression (MR) method was applied for development of 3D-QSAR model. The descriptors chosen were E_337, S_335, E_832, E_424, S_151 and E_721 belonging to steric and electrostatic field energy of interactions with the numbers representing their respective spatial grid points. In this model, no hydrophobic descriptors were selected in the final model. The 3D QSAR model obtained is:

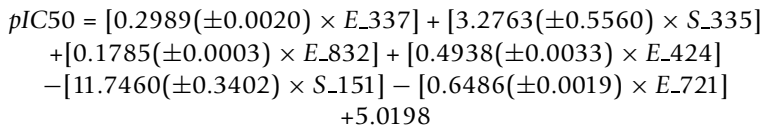

Each descriptor is associated with a numerical coefficient and its error while the last single numerical value is the regression coefficient. Internal and external validation of the developed model was carried out using the LOO method by calculating statistical parameters and meeting critical requirements for a model to be robust. The statistical parameters obtained for this model included correlation coefficient $r^{2}(0.9751)$, cross-validated correlation coefficient $\mathrm{q}^{2}(0.9491)$, predicted correlation coefficient pred_ $r^{2}(0.9525)$, low standard error value, $r^{2}$ se $(0.0966)$, $\mathrm{q}^{2} \_$se $(0.1380)$ andpred_ $\mathrm{r}^{2}$ _se $(0.1282)$ which confirm the 


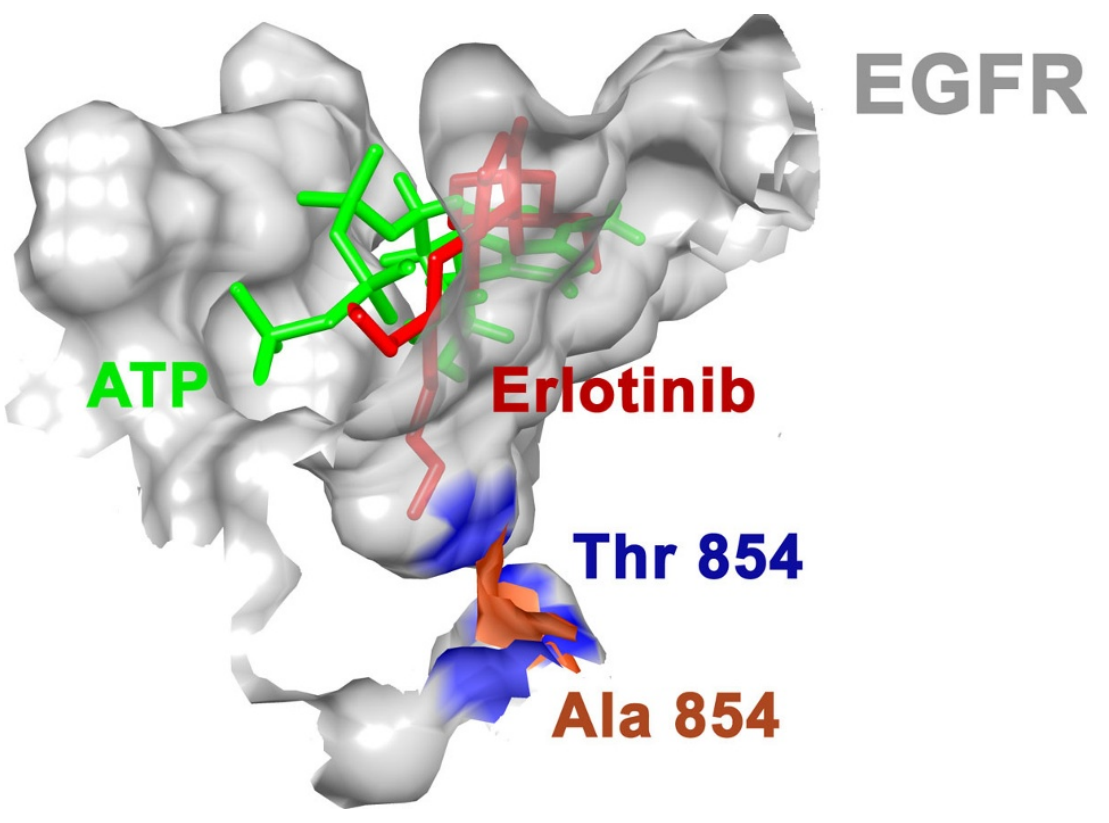

Figure 4 Change in erlotinib binding site due to T854A mutation.

model to be robust. Along with this, high value of F-test (130.3822) implied that the developed QSAR model is 99\% statistically valid with 1 in 10000 chance of failure. There are other important statistical parameters such as Z-scores for $\mathrm{r}^{2}, \mathrm{q}^{2}$ and pred_r $\mathrm{r}^{2}$ which are also important for QSAR model validation. Zscore_r ${ }^{2}$ of 6.7926 implies a $100 \%$ area under the normal curve, Zscore_q ${ }^{2}$ of 4.3671 implies a $99.99 \%$ area under the normal curve and Zscore_pred_r ${ }^{2}$ of 1.6521 implies a $95.0743 \%$ area under the normal curve. These percentages indicate that the respective scores are near the mean ' $\mu$ ' thus validating the model's statistical robustness. A parameter $\mathrm{p}$-value for each of $\mathrm{r}^{2}, \mathrm{q}^{2}$ and pred_r ${ }^{2}$ was also obtained to be statistically significant with values $0.0001,0.0001$ and 0.09 respectively.

The robustness of the model can also be validated by radar and fitness plots. The fitness plot (Figure 5a) shows the extent of variation between the actual and predicted inhibitory activities of the thiazolyl-pyrazoline derived compounds. The radar plots (Figure S2 (a,b); Additional file 1 ) express the quality of the $3 \mathrm{D}-\mathrm{QSAR}$ model by the extent of overlap between the actual value (blue) and predicted activity (red) lines. The contribution plot for each descriptor (Figure S2(c); additional file 1) specifies contribution of the properties that should be present in the lead compound for improving its inhibitory activity. Descriptors with positive contribution enhance the inhibitory activity of the lead compound whereas those with negative contribution reduce the same. Positive contribution for electrostatic descriptor shows a requirement of electropositive group at the substitution site and an electronegative group in case of negativeshi contribution.

The grid points E_337, E_832, S_335 and E_424 had a positive contribution $(8.087 \%, 17.767 \%, 5.291 \%$ and $13.366 \%$ respectively) in the developed 3D-QSAR model against EGFR, while the descriptors S_151 and E_463 show negative contribution of $24.048 \%$ and $31.442 \%$ respectively. The grid points can be seen in Figure 1b. Steric descriptors represent the class of bulk descriptors which describe both size and shape of the molecules and fragments. Thus, positive contribution of a steric descriptor at specific grid point indicates the importance of a bulky group at that position. The value for each descriptor and predicted inhibitory activity for the dataset is mentioned in Table S2 (additional file 1).

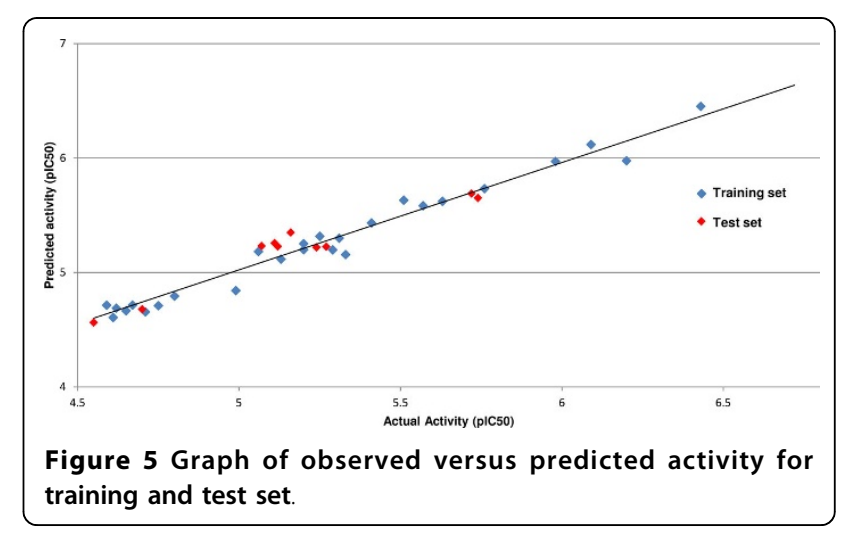


The second class of descriptors, electrostatic descriptors give the importance of electronegative and electropositive groups at a particular site. Electrostatic descriptors with positive contribution imply the significance of presence of electropositive groups while those with negative contribution signify the importance of presence of electronegative groups.

\section{Activity prediction of ZINC libraries using developed 3D-QSAR model}

A total of 0.2 million natural compounds from ZINC library were screened and the highest predicted activity was observed to be 13.436 with 195 compounds having predicted activity above 8 and extrapolation between -1 and 1 . We report the top two compounds with highest predicted activity. The first compound 7-hydroxy-3-(4methoxyphenyl)-8-[(4-methylpiperazin-1-yl)methyl]-4Hchromen-4-one [ZINC ID: 20391511] (HCO) had a predicted activity (pIC50) of 13.44 while the second compound $\mathrm{N}-(2-(1 \mathrm{H}-$ indol-3-yl)ethyl $)-2-((8-o x o-8 H-b e n z o[c]$ indolo[3,2,1-ij][1, 5]naphthyridin-12-yl)oxy)propanamide [ZINC ID: 08792354] (NOP) possessed a predicted activity value of 11.92 (Figure 6). The QSAR model generated was also used to predict the inhibitory activity of a second generation drug, BIBW2992, as reported by Bean et al as a positive control [8]. It was observed that $\mathrm{HCO}$ and NOP possessed better predicted inhibitory activity than BIBW2992 (4.3). Values of top 10 ZINC compounds with their predicted activity can be seen in Table 1 .

\section{Docking analysis of HCO and NOP to both WT and T854A structures}

Both compounds ( $\mathrm{HCO}$ and NOP) with highest predicted inhibitory activity against WT were docked with

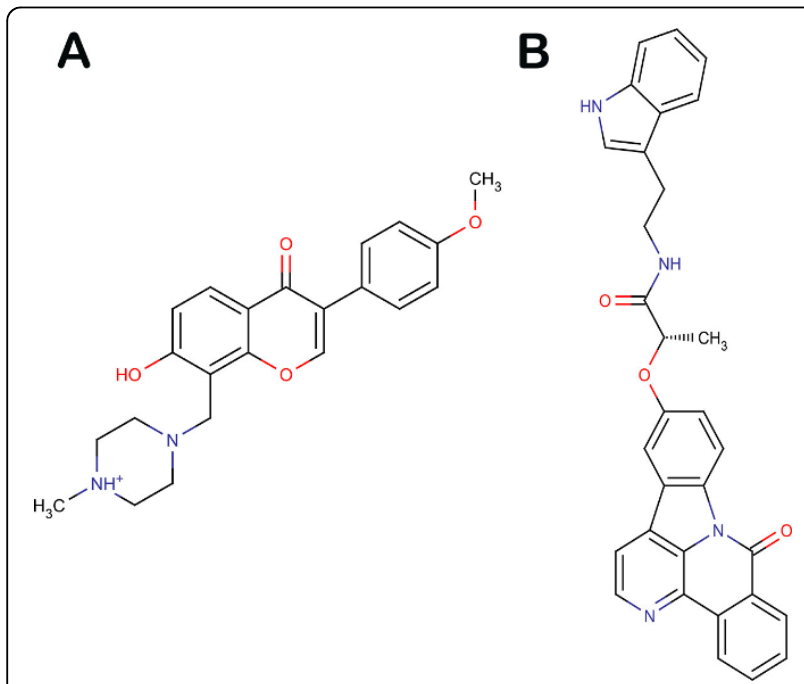

Figure 6 Structure of top (a) HCO and (b) NOP.
Table 1. Predicted activity value (pIC50) of top ten ZINC compounds

\begin{tabular}{cccc}
\hline S.No. & ZINC ID & Predicted Activity & Extrapolation \\
\hline 1 & ZINC20391511 & 13.436 & -0.22 \\
2 & ZINC08792354 & 11.92 & 0.104 \\
3 & ZINC34105774 & 11.075 & 0.232 \\
4 & ZINC12892580 & 9.957 & -0.373 \\
5 & ZINC11865797 & 9.883 & 0.314 \\
6 & ZINC68604752 & 9.68 & -0.364 \\
7 & ZINC08877152 & 9.513 & 0.34 \\
8 & ZINC70700724 & 9.295 & -0.051 \\
9 & ZINC33832195 & 9.142 & -0.462 \\
10 & ZINC41669357 & 8.92 & 0.342 \\
\hline
\end{tabular}

WT and T854A structures. The first compound HCO showed a binding affinity of $-13.025 \mathrm{~kJ} / \mathrm{mol}$ with WT while showing a better binding affinity of $-16.485 \mathrm{~kJ} / \mathrm{mol}$ with T854A structure. The second compound NOP also showed a better binding affinity to T854A $(-8.598 \mathrm{~kJ} / \mathrm{mol})$ than WT $(-8.037 \mathrm{~kJ} / \mathrm{mol})$. The results are summarised in table 2. Thus these compounds can be considered as lead compounds against both WT and T854A structures.

\section{Conclusion}

In the present study, we performed molecular dynamics simulations on both wild-type (WT) and mutant (T854A) structures of EGFR to analyse the structural changes brought about by missense SNP resulting in T854A mutation. A 3D-QSAR model was developed using 38 thiazolyl-pyrazoline derivatives against WT which was then used to screen ZINC libraries by predicting their inhibitory activity (pIC50). The top two compounds were docked against WT and T854A structures. These compounds can be considered as lead drug candidates against both WT and mutant (T854A). The results indicate stability loss observed in RMSD, RMSF, Rg and SASA analysis. Thus it can be said that WT structure becomes more flexible upon mutation (T854A) which brings about changes in the binding site of erlotinib thus reducing its binding affinity and rendering the mutated protein to become drug resistant while maintaining its functionality. This was further supported by results obtained in PCA analysis. This generates the need to develop drugs that inhibit both WT and mutant proteins. We report two novel compounds (HCO and NOP) which

Table 2. Binding affinity of HCO and NOP with WT and T854A mutant structures

\begin{tabular}{ccc}
\hline Compound & \multicolumn{2}{c}{ Score $(\mathbf{k J} / \mathrm{mol})$} \\
& WT & T854A \\
\hline HCO & -13.025 & -16.485 \\
NOP & -8.037 & -8.598 \\
\hline
\end{tabular}


have high predicted inhibitory activity against WT and high binding affinity against both WT and T854A mutant structure. Since these compounds possess better predicted inhibitory activity than BIBW2992 a known second-generation EGFR inhibitor for T854A change, they can be considered for further experimental validation as potent lead compounds. We present a comprehensive view of the correlation between the structure and inhibitory activity of thiazolyl-pyrazoline derived molecules. This study advances the use of thiazolyl-pyrazoline moiety as anti-cancer. Results of this study will also prove to be useful in designing potent anti-tumorals based on EGFR TK inhibition to further develop drugs against cancer.

\section{Additional material}

Additional file 1: This file includes the following figures and tables Figure S1: Graphs showing (a) solvent accessible surface area (SASA) (b) Hydrogen bonds and (c) Total energy of wild-type (blue) and mutant (T854A) (red) protein. Figure S2: Depicting radar plots for (a) training set (b) test set and (c) contribution plot for 3D descriptors. Table S1: Details of thiazolyl-pyrazoline derived compounds along with their actual activity value against WT EGFR. Table S2: Values for descriptors and predicted activity value of thiazolyl-pyrazoline derivatives.

\section{Competing interests}

The authors declare that they have no competing interests.

\section{Authors' contributions}

SG, SJ and AS designed the methods and experimental setup. SG and SJ carried out the work. All the four authors wrote the manuscript.

\section{Acknowledgements}

AG is thankful to Jawaharlal Nehru University for usage of all computational facilities. AG is grateful to University Grants Commission, India for the Faculty Recharge Position.

\section{Declarations}

AG would like to acknowledge financial support from Department of Science and Technology, Government of India towards publication of this article.

This article has been published as part of BMC Genomics Volume 16 Supplement 5, 2015: Proceedings of the 10th International Conference of the Brazilian Association for Bioinformatics and Computational Biology ( $X$ Meeting 2014). The full contents of the supplement are available online at http://www.biomedcentral.com/bmcgenomics/supplements/16/S5.

\section{Authors' details}

'Department of Bioscience and Biotechnology, Banasthali University, Tonk, Rajasthan, India - 304022. ${ }^{2}$ School of Biotechnology, Jawaharlal Nehru University, New Delhi, India - 110067.

Published: 26 May 2015

\section{References}

1. Voldborg BR, Damstrup L, Spang-Thomsen M, Poulsen HS: Epidermal growth factor receptor (EGFR) and EGFR mutations, function and possible role in clinical trials. Ann Oncol 1997, 8(12):1197-1206

2. Kumar A, Petri ET, Halmos B, Boggon TJ: Structure and clinical relevance of the epidermal growth factor receptor in human cancer. J Clin Oncol 2008, 26(10):1742-1751.
3. Shigematsu $H$, Lin L, Takahashi T, Nomura M, Suzuki M, Fong KM, et al: Clinical and biological features associated with epidermal growth factor receptor gene mutations in lung cancers. J Natl Cancer Inst 2005, 97(5):339-346.

4. Lynch TJ, Bell DW, Sordella R, Gurubhagavatula S, Okimoto RA, Brannigan BW, et al: Activating mutations in the epidermal growth factor receptor underlying responsiveness of non-small-cell lung cancer to gefitinib. N Engl J Med 2004, 350(21):2129-2139.

5. Gazdar AF, Minna JD: Deregulated EGFR signaling during lung cancer progression: mutations, amplicons, and autocrine loops. Cancer Prev Res (Phila) 2008, 1(3):156-160.

6. Tokumo M, Toyooka S, Kiura K, Shigematsu H, Tomii K, Aoe M, et al: The relationship between epidermal growth factor receptor mutations and clinicopathologic features in non-small cell lung cancers. Clin Cancer Res 2005, 11(3):1167-1173.

7. Avizienyte $E$, Ward R, Garner A: Comparison of the EGFR resistance mutation profiles generated by EGFR-targeted tyrosine kinase inhibitors and the impact of drug combinations. Biochem J 2008, 415(2):197-206.

8. Bean J, Riely GJ, Balak M, Marks JL, Ladanyi M, Miller VA, Pao W: Acquired resistance to epidermal growth factor receptor kinase inhibitors associated with a novel T854A mutation in a patient with EGFR-mutant lung adenocarcinoma. Clinical Cancer Res 2008, 14(22):7519-7525.

9. Tyagi C, Gupta A, Goyal S, Dhanjal JK, Grover A: Fragment based group QSAR and molecular dynamics mechanistic studies on arylthioindole derivatives targeting the $\alpha-\beta$ interfacial site of human tubulin. $B M C$ Genomics 2014, 15(Suppl 9):S3.

10. Tyagi C, Grover S, Dhanjal JK, Goyal S, Goyal M, Grover A: Mechanistic insights into mode of action of novel natural cathepsin $L$ inhibitors. $B M C$ Genomics 2013, 14(Suppl 8):S10.

11. Cramer RD, Patterson DE, Bunce JD: Comparative molecular field analysis (CoMFA). 1. Effect of shape on binding of steroids to carrier proteins. Journal of the American Chemical Society 1988, 110(18):5959-5967.

12. Akamatsu M: Current state and perspectives of 3D-QSAR. Curr Top Med Chem 2002, 2(12):1381-1394.

13. Dhanjal JK, Grover S, Sharma S, Singh A, Grover A: Structural insights into mode of actions of novel natural Mycobacterium protein tyrosine phosphatase B inhibitors. BMC Genomics 2014, 15(Suppl 1):S3.

14. Saxena N, Katiyar SP, Liu Y, Grover A, Gao R, Sundar D, et al: Molecular interactions of $\mathrm{BCl}-2$ and $\mathrm{BCl}-\mathrm{xL}$ with mortalin: identification and functional characterization. Biosci Rep 2013, 33(5):797-806.

15. Dhiman H, Dhanjal JK, Sharma S, Chacko S, Grover S, Grover A: Resisting resistant $<\mathrm{i}>$ Mycobacterium tuberculosis</i> naturally: Mechanistic insights into the inhibition of the parasite's sole signal peptidase Leader peptidase B. Biochemical and biophysical research communications 2013, 433(4):552-557.

16. Soni S, Tyagi C, Grover A, Goswami SK: Molecular modeling and molecular dynamics simulations based structural analysis of the SG2NA protein variants. BMC Research Notes 2014, 7:446.

17. Lv PC, Li DD, Li QS, Lu X, Xiao ZP, Zhu HL: Synthesis, molecular docking and evaluation of thiazolyl-pyrazoline derivatives as EGFR TK inhibitors and potential anticancer agents. Bioorg Med Chem Lett 2011, 21(18):5374-5377.

18. Bernstein FC, Koetzle TF, Williams GJ, Meyer EF, Brice MD, Rodgers JR, et al: The protein data bank. European Journal of Biochemistry 1977, 80(2):319-324.

19. Maestro S: Version 9.2. LLC, New York; 2011

20. Schrödinger L: SCHRODINGER SUITE 2008. Maestro Version 2008, 8.

21. Sastry GM, Adzhigirey M, Day T, Annabhimoju R, Sherman W: Protein and ligand preparation: parameters, protocols, and influence on virtual screening enrichments. J Comput Aided Mol Des 2013, 27(3):221-234.

22. Van Der Spoel D, Lindahl E, Hess B, Groenhof G, Mark AE, Berendsen HJ: GROMACS: fast, flexible, and free. J Comput Chem 2005, 26(16):1701-1718.

23. van der Spoel D, van Maaren PJ, Caleman C: GROMACS molecule \& liquid database. Bioinformatics 2012, 28(5):752-753.

24. Goyal M, Grover S, Dhanjal JK, Goyal S, Tyagi C, Chacko S, Grover A: Novel Natural Structure Corrector of ApoE4 for Checking Alzheimer's Disease: Benefits from High Throughput Screening and Molecular Dynamics Simulations. BioMed Research International 2013, 2013.

25. Goyal S, Grover S, Dhanjal JK, Goyal M, Tyagi C, Chacko S, Grover A: Mechanistic insights into mode of actions of novel oligopeptidase $B$ inhibitors for combating leishmaniasis. J Mol Model 2014, 20(3):2099. 
26. Mongan J: Interactive essential dynamics. J Comput Aided Mol Des 2004, 18(6):433-436.

27. Goyal S, Dhanjal JK, Tyagi C, Goyal M, Grover A: Novel Fragment-Based QSAR Modeling and Combinatorial Design of Pyrazole-Derived CRK3 Inhibitors as Potent Antileishmanials. Chem Biol Drug Des 2014, 84(1):54-62

28. Goyal M, Grover S, Dhanjal JK, Goyal S, Tyagi C, Grover A: Molecular modelling studies on flavonoid derivatives as dual site inhibitors of human acetyl cholinesterase using 3D-QSAR, pharmacophore and high throughput screening approaches. Medicinal Chemistry Research 2014, 23(4):2122-2132

29. Goyal M, Dhanjal JK, Goyal S, Tyagi C, Hamid R, Grover A: Development of dual inhibitors against Alzheimer's disease using fragment-based QSAR and molecular docking. BioMed Research International 2014, 2014.

30. Spessard GO: ACD Labs/LogP dB 3.5 and ChemSketch 3.5. Journal of Chemical Information and Computer Sciences 1998, 38(6):1250-1253.

31. Bhadoriya KS, Sharma MC, Jain SV, Kad SA, Raghuvanshi D: QSAR Studies of Fused 5, 6-Bicyclic Heterocycles as $y$-Secretase Modulators. J Pharm Res 2012, 5(8):4127-4132.

32. Afantitis A, Melagraki G, Sarimveis H, lgglessi-Markopoulou O, Kollias G: A novel QSAR model for predicting the inhibition of CXCR3 receptor by 4-N-aryl-[1,4] diazepane ureas. Eur J Med Chem 2009, 44(2):877-884.

33. Golbraikh A, Tropsha A: Predictive QSAR modeling based on diversity sampling of experimental datasets for the training and test set selection. Mol Divers 2002, 5(4):231-243.

34. Golbraikh A, Tropsha A: Beware of $q^{2}$. Journal of Molecular Graphics and Modelling 2002, 20(4):269-276.

35. Friesner RA, Banks JL, Murphy RB, Halgren TA, Klicic JJ, Mainz DT, et al: Glide: a new approach for rapid, accurate docking and scoring. 1. Method and assessment of docking accuracy. Journal of Medicinal Chemistry 2004, 47(7):1739-1749.

36. Halgren TA, Murphy RB, Friesner RA, Beard HS, Frye LL, Pollard WT, Banks JL: Glide: a new approach for rapid, accurate docking and scoring. 2. Enrichment factors in database screening. Journal of Medicinal Chemistry 2004, 47(7):1750-1759.

37. Goyal S, Grover S, Dhanjal JK, Tyagi C, Goyal M, Grover A: Group-based QSAR and molecular dynamics mechanistic analysis revealing the mode of action of novel piperidinone derived protein-protein inhibitors of p53-MDM2. Journal of Molecular Graphics and Modelling 2014, 51:64-72.

38. Friesner RA, Murphy RB, Repasky MP, Frye LL, Greenwood JR, Halgren TA, et al: Extra Precision Glide: Docking and Scoring Incorporating a Model of Hydrophobic Enclosure for Protein-Ligand Complexes. Journal of Medicinal Chemistry 2006, 49(21):6177-6196.

doi:10.1186/1471-2164-16-S5-S8

Cite this article as: Goyal et al:: Structural investigations of T854A mutation in EGFR and identification of novel inhibitors using structure activity relationships. BMC Genomics 2015 16(Suppl 5):S8.

\section{Submit your next manuscript to BioMed Central and take full advantage of:}

- Convenient online submission

- Thorough peer review

- No space constraints or color figure charges

- Immediate publication on acceptance

- Inclusion in PubMed, CAS, Scopus and Google Scholar

- Research which is freely available for redistribution

Submit your manuscript at www.biomedcentral.com/submit
C Biomed Central 